OPEN ACCESS

Edited by:

Jianying Gu,

College of Staten Island

United States

Reviewed by:

Chang-Ro Lee

Myongji University, South Korea Andrés Esteban Marcoleta,

University of Chile, Chile

*Correspondence:

Jinbo Liu

liulab2019@163.com

${ }^{\dagger}$ These authors have contributed equally to this work

Specialty section:

This article was submitted to

Evolutionary and Genomic

Microbiology,

a section of the journal

Frontiers in Microbiology

Received: 09 July 2020

Accepted: 14 October 2020

Published: 20 November 2020

Citation:

Tang M, Kong $X$, Hao J and Liu J

(2020) Epidemiological

Characteristics and Formation Mechanisms of Multidrug-Resistant Hypervirulent Klebsiella pneumoniae.

Front. Microbiol. 11:581543.

doi: 10.3389/fmicb.2020.581543

\section{Epidemiological Characteristics and Formation Mechanisms of Multidrug-Resistant Hypervirulent Klebsiella pneumoniae}

\author{
Miran Tang ${ }^{1 \dagger}$, Xin Kong ${ }^{2 \dagger}$, Jingchen $\mathrm{HaO}^{2}$ and Jinbo Liu ${ }^{2 *}$ \\ ${ }^{1}$ Clinical Laboratory Department, Santai People's Hospital, Mianyang, China, ${ }^{2}$ Department of Laboratory Medicine, Affiliated \\ Hospital of Southwest Medical University, Luzhou, China
}

Multi-drug resistance (MDR) and hypervirulence (hv) were exhibited by different wellseparated Klebsiella pneumoniae lineages in the past, but their convergence clonesMDR-hypervirulent $K$. pneumoniae (HvKPs) - both highly pathogenic and resistant to most available antibiotics, have increasingly been reported. In light of the clonal lineages and molecular characteristics of the studied MDR-HvKP strains found in the literature since 2014, this review discusses the epidemiology of MDR-HvKPs, in particular summarizing the three general aspects of plasmids-associated mechanisms underlying the formation of MDR-HvKPs clones: MDR-classic K. pneumoniae (cKPs) acquiring hv plasmids, hvKPs obtaining MDR plasmids, and the acquisition of hybrid plasmids harboring virulence and resistance determinants. A deeper understanding of epidemiological characteristics and possible formation mechanisms of MDR-HvKPs is greatly needed for the proper surveillance and management of this potential threat.

Keywords: Klebsiella pneumoniae, multi-drug resistance, hypervirulent, epidemiology, formation mechanism, plasmid, horizontal gene transfer, mobile genetic elements

\section{INTRODUCTION}

Klebsiella pneumoniae is a clinically relevant opportunistic pathogen that causes a wide range of infections. Hypervirulent K. pneumoniae (hvKPs) and "classic" K. pneumoniae (cKPs) are two different variants of $K$. pneumoniae (Bialek-Davenet et al., 2014). The former are usually hypermucoviscous (HM) and are clinically characterized by their abilities to cause life-threatening invasive community-acquired infections, like entophthalmias and liver abscesses, in a healthy population (Shon et al., 2013). Fortunately, the majority of them have retained susceptibility to multiple commonly used antimicrobial agents except for ampicillin. The latter generally behave as opportunistic avirulent pathogens, causing nosocomial infections in hospitalized patients. Unfortunately, they have a propensity to acquire multiple resistant determinants, such as extendedspectrum $\beta$-lactamases (ESBLs) and/or carbapenemase, to be multi-drug resistant (MDR) strains making treatment more difficult (Navon-Venezia et al., 2017).

In the past, virulence and antibiotic-resistance have evolved separately in these two distinct K. pneumoniae clonal groups (Bialek-Davenet et al., 2014). However, in the face of antibiotic selection pressure, more and more isolates with combined hypervirulence (hv) and MDR have been 
detected and have reached public attention as "superbugs" with the possibility of causing untreatable invasive infections. To better understand such strains, this review focuses on summarizing and discussing the epidemiological characteristics and the possible formation mechanisms of them.

\section{PATHOGEN AND EPIDEMIOLOGY}

Over the past years, a "reference/standard" genotypic/phenotypic marker for hvKP has been lacking, and a positive string test indicating $\mathrm{HM}$ has been regarded as an important in vitro parameter for hvKP identification in some early studies (Yao et al., 2015). Yet, as research progressed, several controversies regarding the association of $\mathrm{HM}$ phenotype and hv have been raised. HM is not exclusive to hv, on the contrary, hvKP does not absolutely present HM (Carlos Catalan-Najera et al., 2017). In this review, studies involving hv (well-defined by virulence-related assays) or HM (positive for string test) strains were included.

Klebsiella pneumoniae has experienced the evolution of thirdgeneration cephalosporin-, carbapenem-, and even polymyxinresistance. Harboring a wide range of $\beta$-lactamases results in third-generation cephalosporin-resistance in K. pneumoniae isolates. Acquired resistance to carbapenems can be conferred by carbapenemase production, such as KPC, OXA, and MBLs which include NDM, VIM, IMP, as well as SIM (Tzouvelekis et al., 2012). Then, the prevalence of carbapenem-resistant bacteria has led to the use of polymyxins as a last-therapy option to treat associated infections, which has resulted in the emergence of polymyxinsresistant $K$. pneumoniae. This review includes literature about any kind of MDR phenotypes hvKPs.

We performed an exhaustive search of PubMed, MEDLINE, Web of Science, EMBASE, CNKI, and Wanfang database for English-language literature published before January, 2020, with the following search strategy ("hypervirulence"[All Fields] OR "hypervirulent"[All Fields] OR "hypermucoviscous"[All Fields]) AND ("Klebsiella pneumoniae"[MeSH Terms] OR "Klebsiella pneumoniae"[All Fields]) AND ("resistance"[All Fields] OR "resistant"[All Fields]), then summarized and classified these papers according to the country or region, STs, capsule types, plasmid replicon types, resistance loci, and formation mechanisms in Table 1, which we will refer to frequently throughout this section.

From Table 1, we can see that MDR-HvKPs have mainly been detected since 2014 and have become research hotspot. All studies since 2014 involving both MDR and hv K. pneumoniae isolates account for a total of 47. China (including Taiwan) accounts for 33, and the remaining 13 are from other Asian countries (three from India, one from Japan, and one from Iran), Europe (two from the United Kingdom, one from France, one from Italy, and one from Norway), North America (one from United States), and South America (one from Brazil and another from Argentina). In our opinion, such geographical distribution is attributed to the prevalence characteristics of MDR and hv-KP strains around the world. For example, the high prevalence of both hvKPs and MDR-KPs in China and the significant proportions of incidence of MDR-KPs in other Asian countries (Lee et al., 2016, 2017) may contribute to the majority of reports about MDR-HvKPs coming from these regions.

By comparing the allelic sequences of seven housekeeping genes, multi-locus sequence typing (MLST) can structure K. pneumoniae populations into lineages, which are typically referenced by their sequence types (STs; e.g., ST11). The common MDR-KP strains are strongly linked to particular clonal complexes (CCs), like CC258, comprising ST258, ST11, ST512, ST340, ST437, etc. (Schweizer et al., 2019), CC15 and CC14, while hvKPs mainly belong to ST23 for the K1 capsular serotype and to ST86, ST65, and ST25 for K2 (Bialek-Davenet et al., 2014). Hence, the genetic backgrounds of the isolates, i.e., the strains stem from whether resistant or virulent lineage, can be determined by STs. MDR-HvKPs showed various STs in the literature (Table 1). Among them, the most prevalent ST was the KPC-producing CRKP highly related ST11, followed by the dominant hvKP lineage ST23. This was in accordance with the fact that ST11 and ST23 are predominant among MDR-KP and hvKP strains, respectively (Bialek-Davenet et al., 2014). In addition, STs showed different region distributions. In China, ST23 accounted for about $69.57 \%(16 / 23)$ in all hvKPs and ST11 took on almost the same high proportion $77.27 \%(51 / 66)$ in all MDR-KPs, respectively, which was consistent with the fact that both KPC-KP and hvKP are prevalent in China (Lee et al., 2016, 2017). While in other countries, except for India and Brazil, ST11 was almost undetected; instead, STs representing a virulent clone, such as ST23, ST25, and ST86, were more easily detected, which was in line with the fact that hvKP has increasingly prevailed in Europe and the Americas in recent years (Cubero et al., 2016; Lee et al., 2017).

\section{POSSIBLE FORMATION MECHANISMS}

Bacterial phenotypic changes including resistance and virulence acquisition are mainly driven by horizontal gene transfer (HGT) (in addition to chromosomal mutations) mediated by mobile genetic elements (MGEs), such as plasmids, insertion sequences (IS), transposons ( $\mathrm{Tn}$ ), integrons (In) and integrative conjugative elements (ICEs), driving dissemination and co-selection of virulence and resistance genes through genomic rearrangement during their replication or recombination process, mostly in Gram-negative pathogens including K. pneumoniae (Frost et al., 2005). Compared with transformation and transduction, thought to be secondary effects of other biological processes, conjugation plays a most impactful role in HGT due to the transfer of plasmids and ICEs via direct cell-to-cell contact (Pinilla-Redondo et al., 2018). Additionally, in most instances, hv or MDR phenotype selection genes transferred by Tn, In, and IS were mostly accumulated on plasmids followed by plasmid inter bacterial transfer. Consequently, as the most pivotal vectors for conjugation and genes recruitment, plasmids are arguably the most indispensable and essential elements throughout the entire convergence process of hv and MDR genes coming from strains of distinct backgrounds. Accordingly, in this section, we dissect the available mechanism studies apart from case reports 
TABLE 1 | Lists of papers referenced.

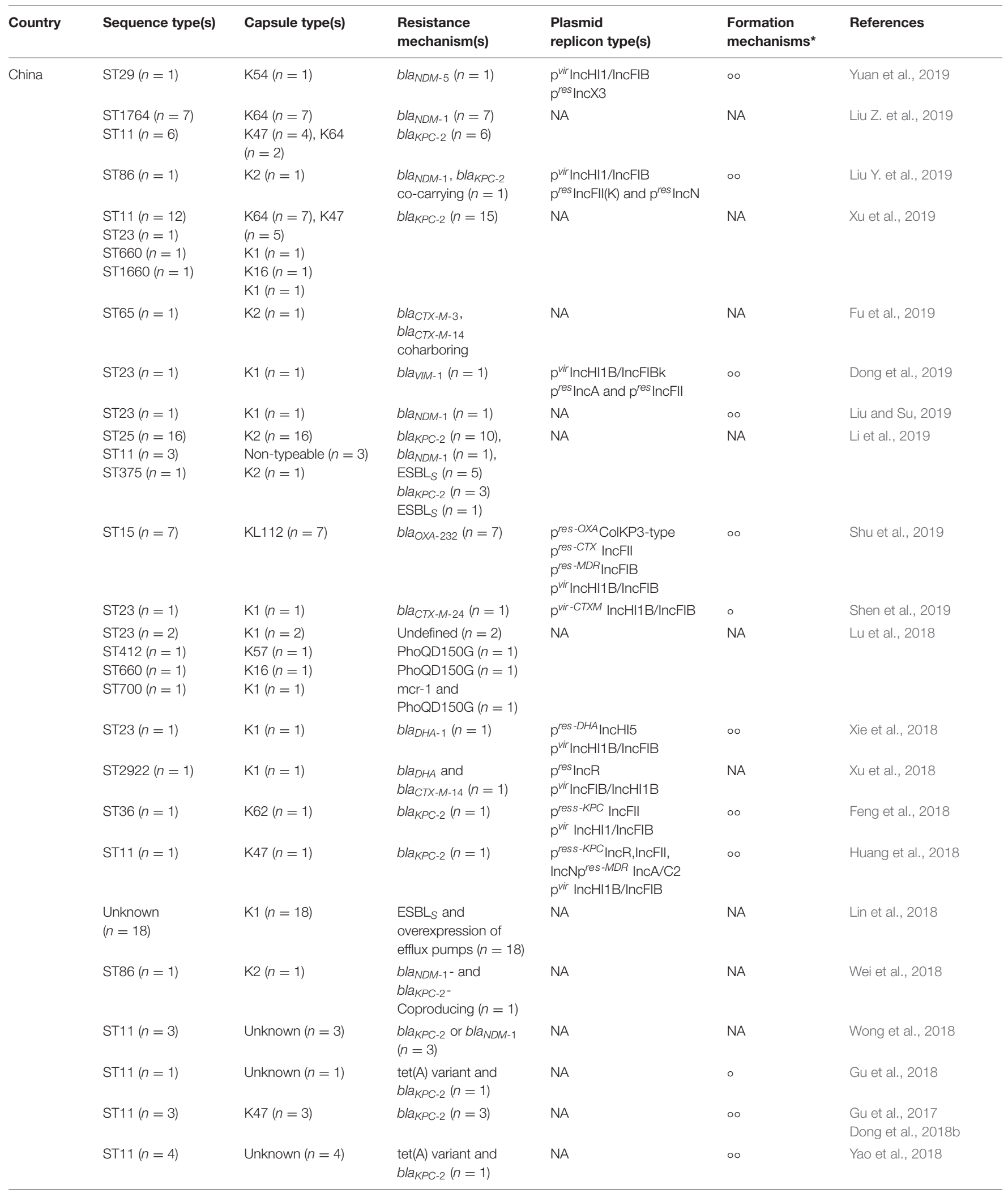


TABLE 1 | Continued

\begin{tabular}{|c|c|c|c|c|c|c|}
\hline Country & Sequence type(s) & Capsule type(s) & $\begin{array}{l}\text { Resistance } \\
\text { mechanism(s) }\end{array}$ & $\begin{array}{l}\text { Plasmid } \\
\text { replicon type(s) }\end{array}$ & $\begin{array}{l}\text { Formation } \\
\text { mechanisms* }\end{array}$ & References \\
\hline & $\begin{array}{l}\text { ST11 }(n=16) \\
\text { ST268 }(n=2) \\
\text { ST65 }(n=1) \\
\text { ST692 }(n=1) \\
\text { ST595 }(n=1)\end{array}$ & $\begin{array}{l}\mathrm{K} 20(n=5), \text { non- } \\
\mathrm{T}(n=11) \\
\mathrm{K} 20(n=1), \text { non- } \\
\mathrm{T}(n=1) \mathrm{K} 2(n=1) \\
\text { non- }(n=1) \\
\text { non- } \mathrm{T}(n=1)\end{array}$ & $\begin{array}{l}\text { blakPC-2 and } \\
\text { blaSHV-11 }(n=16) \\
\text { blaKPC-2 and } \\
\text { blaSHV-11 }(n=2) \\
\text { blakPC-2 and } \\
\text { blaSHV-11 }(n=1) \\
\text { blakPC-2 and } \\
\text { blaSHV-11 }(n=1) \\
\text { blakPC-2 }(n=1)\end{array}$ & NA & NA & Zhan et al., 2017 \\
\hline & $\begin{array}{l}\text { ST23 }(n=2) \\
\text { ST268 }(n=3) \\
\text { ST65 }(n=1) \\
\text { ST17 }(n=1) \\
\text { ST420 }(n=1) \\
\text { ST367 }(n=1) \\
\text { ST1658 }(n=1) \\
\text { ST35 }(n=1)\end{array}$ & $\begin{array}{l}\text { K1 }(n=2) \\
\text { K1 }(n=1), \text { K20 } \\
(n=2) \\
\text { K2 }(n=1) \\
\text { Non-typeable } \\
(n=1) \\
\text { K20 }(n=1) \\
\text { K1 }(n=1) \\
\text { K2 }(n=1) \\
\text { Non-typeable } \\
(n=1)\end{array}$ & $\begin{array}{l}\text { SHV-75, CTXM-55, } \\
\text { SHV-11, TEM-1, } \\
\text { CTX-M-like, SHV-148, } \\
\text { CTX-M-14, } \\
\text { TEM-53 }(n=11)\end{array}$ & NA & NA & Zhang Y.W. et al., 2016 \\
\hline & $\begin{array}{l}\text { ST86 }(n=7) \\
\text { ST37 }(n=6) \\
\text { ST23 }(n=5)\end{array}$ & Unknown & $\mathrm{ESBL}_{S}(n=18)$ & NA & NA & Zhang J. et al., 2016 \\
\hline & ST661 $(n=1)$ & $\mathrm{K} 1(n=1)$ & $\operatorname{mcr}-1(n=1)$ & NA & NA & Gu et al., 2016 \\
\hline & ST14 $(n=1)$ & $\mathrm{K} 2(n=1)$ & bla $_{N D M-5}(n=1)$ & NA & NA & Liu et al., 2016 \\
\hline & $\operatorname{ST} 11(n=1)$ & $\mathrm{K} 1(n=1)$ & bla $_{K P C-2}(n=1)$ & 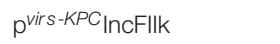 & $\circ$ & Wei et al., 2016 \\
\hline & $\begin{array}{l}\operatorname{ST} 23(n=1) \\
\operatorname{ST} 23(n=1) \\
\operatorname{ST} 1797(n=3)\end{array}$ & $\begin{array}{l}\mathrm{K} 1(n=1) \\
\mathrm{K} 1(n=1) \\
\mathrm{K} 1(n=3)\end{array}$ & $\begin{array}{l}\text { bla }_{K P C-2}(n=1) \\
\text { bla } \\
\text { bla } \\
\text { KPC-2 }(n=1) \\
(n=3)\end{array}$ & $\begin{array}{l}\mathrm{p}^{\text {virs }-K P C} \text { IncHI1B/ IncFIB } \\
\text { NA } \\
\text { NA }\end{array}$ & $\begin{array}{l}\circ \\
\circ \\
\circ\end{array}$ & $\begin{array}{l}\text { Zhang R. et al., } 2015 \\
\text { Dong et al., } 2018 \text { a }\end{array}$ \\
\hline & ST65 $(n=1)$ & $\mathrm{K} 2(n=1)$ & $\begin{array}{l}\text { SHV-11,TEM-53- } \\
\text { producing } \\
\text { ompK35,36 decreased } \\
(n=1)\end{array}$ & NA & NA & Zhang Y.W. et al., 2015 \\
\hline United Kingdom & $\begin{array}{l}\text { ST101 }(n=3) \\
\text { ST383 }(n=3) \\
\text { ST147 }(n=4) \\
\text { ST15 }(n=2) \\
\text { ST48 }(n=1)\end{array}$ & Unknown & $\begin{array}{l}\text { blaOXA-48 and MDR } \\
(n=1), \mathrm{MDR}(n=2) \\
\text { blaOXA-48 }(n=1), \\
\text { bla } \\
(n=1) \\
\text { bla }(n=2), \mathrm{MDR} \\
\text { ble } 1 \text { and MDR } \\
(n=2), \mathrm{MDR}(n=2) \\
\text { blaNDM and MDR } \\
(n=1), \mathrm{MDR}(n=1) \\
\text { bla } \mathrm{NDM}-5 \text { and MDR } \\
(n=1)\end{array}$ & $\begin{array}{l}\mathrm{p}^{\text {vir-MDR-OXA-48 }} \text { and (or) } \\
-\mathrm{NDM} \text { IncFII(K)/IncFIB(K) } \\
\mathrm{p}^{\text {res }-\mathrm{OXA}-48} \text { IncL/M } \\
\mathrm{p}^{\text {res-NDM-1 IncFIB(pQil) }} \\
\mathrm{p}^{\text {vir-NDM-5 IncFIB(Mar) }}\end{array}$ & $\circ$ & $\begin{array}{l}\text { Turton et al., 2017, } \\
2019\end{array}$ \\
\hline Argentina & $\operatorname{ST} 25(n=1)$ & $\mathrm{K} 2(n=1)$ & blakPC-2 $(n=1)$ & NA & NA & Cejas et al., 2019 \\
\hline Japan & ST23 $(n=1)$ & $\mathrm{K} 1(n=1)$ & blalMP-6 $(n=1) q q$ & $\mathrm{p}^{\text {res-IMP-6} I n c N}$ & $\infty$ & Harada et al., 2019 \\
\hline Norway & $\operatorname{ST} 15(n=2)$ & $\mathrm{K} 24(n=2)$ & $\mathrm{ESBL}_{S}(n=2)$ & $\begin{array}{l}\mathrm{p}^{\text {vir-CTXM }} \\
\operatorname{IncFIB}_{K_{1}} / \operatorname{IncFI}_{K_{1}}\end{array}$ & $\circ$ & Lam et al., 2019 \\
\hline Iran & ST23 $(n=5)$ & $\mathrm{K} 1(n=5)$ & blaVIM-2 ( $n=5)$ & $\mathrm{p}^{r e s-V I M-2} \mathrm{IncN}$ & $\infty$ & Tabrizi et al., 2018 \\
\hline United Kingdom & ST23 $(n=1)$ & $\mathrm{K} 1(n=1)$ & $\operatorname{bla}_{N D M-1}(n=1)$ & NA & NA & Roulston et al., 2018 \\
\hline Italy & $\operatorname{ST} 512(n=1)$ & Unknown $(n=1)$ & blaKPC-3 $(n=1)$ & NA & NA & Arena et al., 2017 \\
\hline France & ST86 $(n=1)$ & $\mathrm{K} 2(n=1)$ & bla $_{C T X-M-3}(n=1)$ & $\mathrm{p}^{r e s} \operatorname{IncL} / \mathrm{M}$ & NA & Surgers et al., 2016 \\
\hline India & $\operatorname{ST} 2318(n=1)$ & $\begin{array}{l}\text { Non-typeable } \\
(n=1)\end{array}$ & $\mathrm{ESBL}_{S}(n=1)$ & NA & NA & Shankar et al., 2016b \\
\hline
\end{tabular}


TABLE 1 | Continued

\begin{tabular}{|c|c|c|c|c|c|c|}
\hline Country & Sequence type(s) & Capsule type(s) & $\begin{array}{l}\text { Resistance } \\
\text { mechanism(s) }\end{array}$ & $\begin{array}{l}\text { Plasmid } \\
\text { replicon type(s) }\end{array}$ & $\begin{array}{l}\text { Formation } \\
\text { mechanisms* }\end{array}$ & References \\
\hline India & $\begin{array}{l}\operatorname{ST} 11(n=1) \\
\text { ST43 }(n=1) \\
\text { ST231 }(n=1)\end{array}$ & Unknown & $\begin{array}{l}\text { bla OXA-232, bla }{ }_{O X A-181} \\
\text { bla }_{O X A-1}, \text { bla }_{N D M-1}\end{array}$ & $\begin{array}{l}\text { IncFIA IncFIB IncFII } \\
\text { IncHI1B Col }\end{array}$ & NA & Shankar et al., 2016a \\
\hline India & $\operatorname{ST} 14(n=1)$ & Unknown $(n=1)$ & $\begin{array}{l}\text { Mutation in OmpK36 } \\
(n=1)\end{array}$ & NA & NA & Rafiq et al., 2016 \\
\hline United States & $\operatorname{ST23}(n=1)$ & Unknown $(n=1)$ & $b_{1}{ }_{K P C-2}(n=1)$ & $\mathrm{p}^{r e s-K P C} \mathrm{IncFIA}_{\ln }$ & NA & Cejas et al., 2014 \\
\hline Brazil & $\operatorname{ST11}(n=7)$ & Unknown $(n=7)$ & $\begin{array}{l}\text { bla } \\
\text { blaCTX-2 qnrS1 } \\
\text { ble }(n=7)\end{array}$ & $\left.\mathrm{p}^{\text {ress }-K P C}\right|_{\mid \mathrm{ncF}}$ & NA & Andrade et al., 2014 \\
\hline
\end{tabular}

*Single o represents acquiring hybrid plasmid by KPs, double oo represents acquiring additional plasmid(s), pir by MDR KPs or pres by hvKPs, respectively.

(Table 1) to extract and further expand to three general aspects of plasmids-associated mechanisms underlying the convergence of hv and MDR phenotypes.

\section{MDR-cKPs Acquire Hypervirulence Plasmids}

Virulence plasmid acquisition is an important mechanism for the increased virulence of MDR-cKPs. The best characterized virulence plasmids are the $224 \mathrm{kbp}$ plasmid pK2044 from K1, ST23 strain NTUH-K2044; the 219 kbp plasmid pLVPK from K2, ST86 strain CG43; and the $121 \mathrm{kbp}$ plasmid Kp52.145pII from K2, ST66 strain Kp52.145, on which the virulence-associated loci and genes were highly conservatively organized (Lam et al., 2018). There are some convincing and rational explanations to the comparative rarity of hv plasmids in avirulent MDR-KP clones compared to in hvKPs. First, dominant hv plasmids hosting KP ST23 accounts for only $\leq 2 \%$ of clinical K. pneumoniae isolates in the global range, except for in the Asia-Pacific rim, despite circulating among humans with hv plasmid maintenance for more than 100 years, far longer than the most well-known MDR clones (Wyres et al., 2020), suggesting that the hv plasmids rarely move horizontally and are highly restricted to hvKP clones, i.e., lineage specificity of plasmid distribution. Second, large numbers of plasmids, like hv plasmids in K. pneumoniae, devoid of genes permitting their transfer by conjugation and relying on the transfer function of other plasmids to enable their transfer, are mobilizable but not self-transmissible (Smillie et al., 2010), which radically limits the frequency of the horizontal transfer of hv plasmids. Third, as large low-copy-number plasmids, virulence plasmids contain specific replication and maintenance systems to ensure their transmission to daughter cells of specific genetic backgrounds (Million-Weaver and Camps, 2014), which is reminiscent of the fact that they might often impose fitness costs on other unsuitable host. Despite those restrictive factors, in fact, MDR-cKPs acquiring virulence plasmids have recently been reported. Yao et al. (2018) screened four ST11 CR-HvKP strains from clinical patients in Henan province, China, each of which carried both a KPC-2-encoding and a virulence plasmid. Further sequencing of the virulence plasmid showed high homology to pLVPK. Resembling that, Gu et al. (2018) reported the emergence of a tigecycline- and carbapenem-co-resistant ST11 hvKP isolate from a patient's gut in Zhejiang, China. One of its three plasmids shared high homology with pLVPK and another co-carried $b l a_{K P C-2}$ and $t e t(\mathrm{~A})$. In the Lancet, $\mathrm{Gu}$ et al. reported a fatal outbreak of ST11 CR-HvKP strains in a Chinese hospital. In addition to their intrinsic conjugative MDR plasmids carrying $b l a_{K P C-2}$ genes, the five representative causative strains acquired an additional virulence plasmid that aligned well to most parts of pLVPK (Gu et al., 2017). Subsequently, Dong et al. made the whole genome sequences (WGS) of three ST11 CR-HvKP isolates surveyed in the previous $\mathrm{Gu}$ et al.'s study and five plasmids harbored by each of them. The presence of homologous regions between the virulence plasmid and $b l a_{K P C-2}$-bearing conjugative MDR plasmid suggested that their co-integrated transfer might mediate the transmission of the non-conjugative virulence plasmid from hvKP to ST11 CRKP (Dong et al., 2018b). The main explanation for these facts is that the virulence plasmids are not self-transmissible but often mobilized to access new hosts with the help of other ICEs or conjugative resistance plasmids encoding the conjugation transfer complex in the same host cell (Ramsay and Firth, 2017). Of note, the parental hvKPs, typically susceptible to antimicrobial agents, generally do not originally carry the conjugative resistance plasmid, so in this scenario, the event that hv plasmids are synergistically mobilized by conjugative resistance plasmids in the same host cell to new strains happens after the conjugative resistance plasmids are transferred into hvKPs first, as will be discussed in the next section.

\section{Hv (HM) KPs Obtain Multidrug-Resistance Plasmids}

From the comparatively lower genome's diversity in hvKPs than MDR-KPs, Wyres et al. (2019) inferred that hv clones are less likely to acquire resistance genes than MDR clones are to acquire virulence genes. Nevertheless, evidence seemly shows the opposite. We found that out of all the documents reviewed, the total number of $\mathrm{Hv}$ (HM) KP isolates obtaining MDR plasmids was much more than that of MDR-cKPs acquiring hv plasmids, 111 vs. 87, respectively. (Not absolutely, but it seems to be a doubt). In Zhang R. et al.'s (2015) study, both carbapenems-susceptible HvKP and CR-HvKP strains harbored an $\sim 200-\mathrm{kb}$ virulence plasmid but the latter had acquired two additional plasmids with $b l a_{K P C-2}$ gene located on a transferable 
plasmid. Tabrizi et al. described the emergence of VIM-2encoding K1 ST23 CR-HvKP in an outbreak in Iran. Plasmid analysis revealed a class 1 In carrying $b l a_{V I M-2}$ located on an $\sim 45-\mathrm{kb}$ IncN conjugative plasmid (Tabrizi et al., 2018). As reported by Feng et al. (2018), a bla $a_{K P C-2}$-mediated carbapenemresistant ST36 hvKP clinical isolate had two plasmids, one IncHI1/IncFIB plasmid highly similar to the known pLVPK, another IncFII plasmid carrying $b l a_{K P C-2}$ and proved selftransmissible. Similarly, the first IMP-producing K1 ST23 CRHvKP in Japan carried a pLVPK-like plasmid and an IncN plasmid harboring class 1 In-mediated bla $I M P-6$. According to the fact that $b l a_{I M P-6}$ was, while $r m p A$ was not, detected in the transconjugant, the authors inferred that the bla $a_{I M P-6}$-carrying plasmid was conjugative, but the plasmid carrying virulence gene was not, and they were two individual plasmids (Harada et al., 2019). Liu et al. presented an NDM-1 and KPC-2 coproducing K2 ST86 CR-HvKP strain with four plasmids in China. Apart from an IncHI1/IncFIB virulence plasmid identical to pLVPK, the strain additionally acquired two carbapenemaseproducing plasmids including $b l a_{N D M-1}$-carrying IncN plasmid and IncFIIK plasmid which carried $b l a_{K P C-2}$ and an array of other resistance elements (Liu Y. et al., 2019). Xie et al. delineated a bla $a_{D H A-1}$-carrying IncHI5 plasmid which had a 26-kb accessory region where the bla $a_{D H A-1}$ gene was located upstream of ISCR1 isolated from a K1 ST23 MDR-HvKP strain. Apart from this MDR plasmid, this strain carried another two plasmids, including its virulence plasmid (Xie et al., 2018). In Dong et al. (2019) study, a VIM-1-producing K1 ST23 CR-HvKP strain harbored three plasmids; a virulence plasmid highly homologous to that recovered from other ST23 hvKPs; and a bla $a_{V I M-1}$ bearing plasmid possessing a unique resistance island structure presumably generated by multiple gene mobilization events. Yuan et al. (2019) showed that apart from an IncHI1/IncFIB pLVPK-like plasmid, a bla NDM-5-carrying K54 ST29 CR-HvKP isolated from Sichuan, China, harbored a bla $a_{N D-5}$-carrying IncX3 self-transmissible plasmid. Recent WGS work of an NDM-1-producing K1 ST23 CR-HvKP in China, by Liu and $\mathrm{Su}$ (2019), showed that in addition to a pLVPK-like virulence plasmid, it had a conjugative resistance plasmid carrying a $b l a_{N D M-1}$ and another six types of resistance genes surrounded by ISs. All of these studies were typical examples in which hvKP clones additionally acquired resistance plasmids all proved conjugative. On the theoretical basis that most large resistance plasmids encode their own transfer and are conjugative, we could further speculate from these papers that the surveyed phenotypically convergent strains originally carried a virulence plasmid and thereafter acquired extra resistance plasmids, which are readily transmitted by horizontal transfer between different lineages and species. Nevertheless, if we only consider the strong transfer of resistance plasmids, we cannot explain why the composite strains are still far less than MDR-KP strains. Therefore, we guess that the success of the MDR and hv convergence results from interaction of various positive and negative factors. Clear examples of the positive factors include the following: (i) most resistance plasmids encode all functions needed for their horizontal transfer, such as DNA replication and copy number control functions, mating pair formation genes, and an origin of transfer (oriT) (Pinilla-Redondo et al., 2018), which facilitated their transfer into other strains like hvKPs; (ii) acquisition of antibiotic resistance genes to become MDR-HvKPs promotes adaptive evolution of hvKP clones in an antibiotic environment; (iii) most acquisition of antibiotic resistance will reduce the virulence and fitness of the strain, especially in the absence of antibiotic selection (Durao et al., 2018); while in the era of antibiotics, antibiotic selection pressure promotes plasmids persistence once resistant mutants form; (iv) conjugative resistance plasmids are usually large and have a low-copy-number, which to some extent circumvent use of host material, and as such, has less effect on host fitness than high-copy-number ones; (v) host genetic background might be a pivotal determinant of plasmid fitness. Strain-dependent compensation to the cost of resistance acquisition might occur in hv strains. Intriguingly, not as many strains of concurrent hv and MDR phenotypes, as imagined in the context of so many positive advantages, implied the essential role of negative factors: (i) considerably lower genome diversity and plasticity than that of MDR-KP is a hint that there might be some sort of barrier for hvKP to uptake and/or integrate DNA fragments; (ii) hv strains typically sensitive to antibiotic are selectively killed by antimicrobial agents before they acquire resistance plasmids. Therefore, antibiotic usage creates conditions for the growth and development of MDR-cKP populations rather than producing a noticeable number of MDR-HvKPs. Altogether, we provide explanations for the complex interactions between resistance plasmids and hvKPs, which shed light on the mechanisms of $\mathrm{Hv}$ (HM) KPs obtaining and maintaining MDR plasmids. Notwithstanding Wyres et al.'s (2019) opinion posed at the beginning of this section, that hvKPs are unable or difficult to obtain resistance plasmids, it is possible to state that the genomic traits of hvKPs just weaken the strong transmission kinetics of MDR factors into themselves than into cKPs, producing relatively fewer MDR-hvKPs than MDR-cKPs. Under the interaction of many factors, it is still very easy for hvKPs to obtain resistance plasmids.

\section{Virulence-Resistance Hybrid Plasmids}

Small MGEs, such as Tn, IS, or In, that "hitchhike" on the plasmids, especially resistance plasmids (Pinilla-Redondo et al., 2018), contribute to the capture and dissemination of MDR and/or hv genes whose co-existence on the same plasmid constitutes the perfect mosaic structure-hybrid plasmid. The transfer of the hybrid plasmids into either hvKP or cKP strains form MDR-HvKPs. The possible evolution pathway of MDRHvKPs mediated by the hybrid plasmid is shown in model diagram Figure 1.

Some clear examples have been demonstrated. Zhang et al. reported the emergence of five K1 CR-HvKP strains causing fatal infections in hospital patients in Zhejiang Province, China. The K1 ST23 CR-HvKP70-2 harbored an $~ 200-K p$ plasmid on which bla $a_{K P C}$ and $r m p A$ were located, and this plasmid was not transferred to Escherichia coli. Similarly, bla $a_{K P C-2}$ was detected on two virulence genes-harboring plasmids which were not transferable to $E$. coli in other three genetically related K1 ST1797 isolates (Zhang R. et al., 2015). The non-conjugativity 


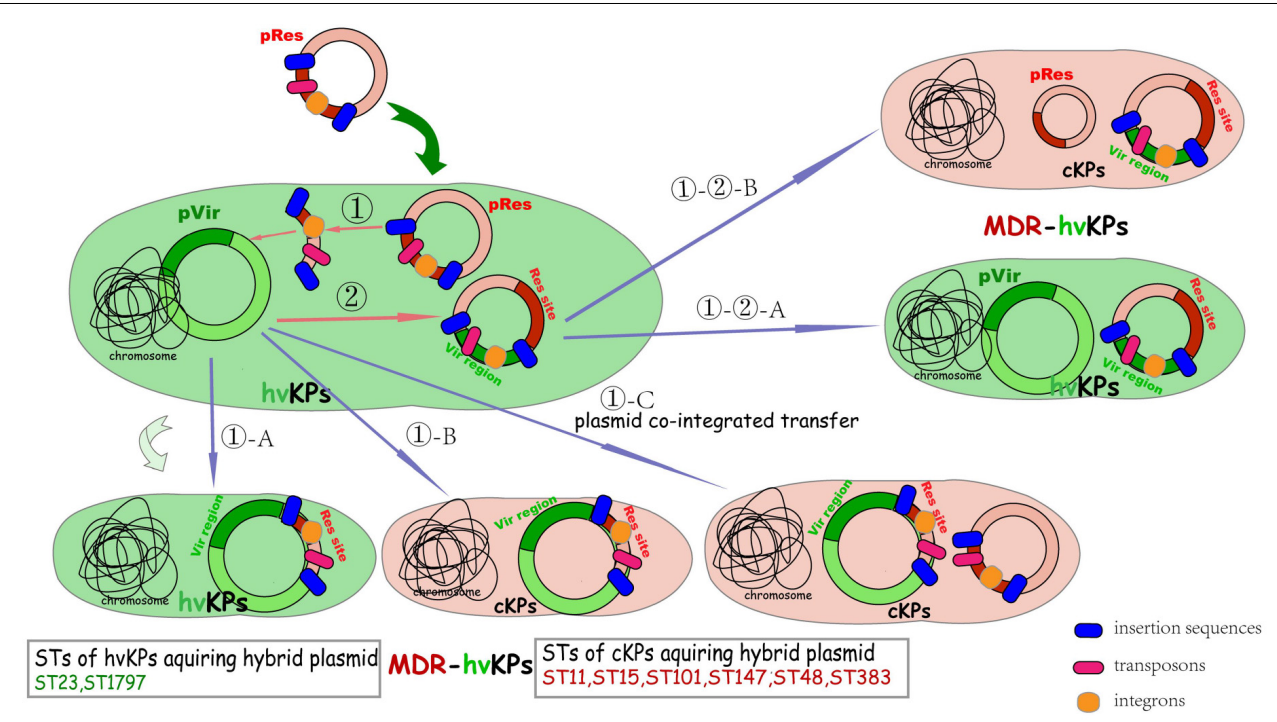

Model diagram of the possible evolution pathway of MDR-hvKPs mediated by hybrid plasmid.

FIGURE 1 | Model diagram of the possible evolution pathway of MDR-hvKPs mediated by a hybrid plasmid. (1) MDR plasmids first transfer into hvKPs, then antimicrobial resistance genes are integrated or transposed into hv plasmid harbored by hvKPs, resulting in the formation of hybrid plasmids with most hv genes-bearing regions and the MDR-hvKPs of hv-associated STs (1)-A). Alternatively, if the genes encoding the self-transfer conjugative system are integrated into the virulence plasmid, together with the resistance determinants, the hybrid plasmids will be conferred self-transmission and conjugativity transfer into any bacterial host including CKPs to become MDR-HvKPs (1)-B). In addition, with the help of other conjugative plasmids this hybrid plasmid can be possibly transferred into other cKPs to form MDR-hvKPs of MDR- or cKP-linked STs (1)-C). (2) If the In, Tn, and (or) Is further carry hv genes from the hv plasmid into other resistance plasmids, hybrid plasmids with most sites of resistance plasmid characteristics are formed. They can be transferred into either hvKPs (1)-(2)-A) or cKPs (1)-(2)-B) to form MDR-hvKPs via their conjugal transfer system.

implied that these hv-MDR plasmids were very likely originally harbored by the hvKPs and carried the hv plasmid backbone, then formed mosaic structures via MGEs-mediated integration with the $b l a_{K P C-2}$-bearing DNA fragment which comes from other conjugative bla $a_{K P C-2}$-carrying resistance plasmid(s) transferred into the hv host before (Figure 1(1)-A). This hypothesis was subsequently proved in Dong et al.'s study where a hybrid plasmid recovered from CR-HvKP strain KP70-2 was found to be almost structurally identical to numerous known hv conferring plasmids harbored by other hvKP strains, except for an extra MDR-encoding region flanked by two copies of IS26 in the same orientation and MGEs-mediated resistance genes $d f r A 14$ and $b l a_{K P C-2}$. The authors concluded that multiple IS elements were responsible for the integration of the MDR region into the virulence plasmid (Dong et al., 2018a). Similarly, the complete genome of an ESBL-producing K1 ST23 MDR-HvKP showed that the strain obtained a rare plasmid harboring virulence and bla comparative analysis illustrated that this plasmid exhibited high similarity with three IncHI1B/IncFIB virulent plasmids retrieved from the GenBank, except for a unique bla harboring region. Further exploration proved that following the IS-mediated bla $a_{C T X-M-24}$ gene insertion, into the conserved virulence plasmid backbone region, the hybrid plasmid formed (Shen et al., 2019). In conclusion, these studies suggested that hv strains are capable of acquiring MDR determinants through the integration of the MDR region mediated by MGEs, like
Tn, In, and seemly dominant IS, into its intrinsic virulence plasmid. The integration of additional resistance elements into the virulence plasmids of hvKPs constitutes perfect mosaic plasmids possessing dual characteristics of conserved virulent regions and newly acquired $\mathrm{MDR}$-encoding sites, but maybe not conjugative (Figure 1 1 -A).

However, if the genes encoding a self-transfer conjugative system are integrated into the virulence plasmid together with the resistance determinants, the hybrid plasmids will be conferred self-transmission and conjugativity. Alternatively, with the help of other conjugative plasmids, this hybrid plasmid could possibly be mobilized to transfer. Hence, if the fitness cost brought into the host bacteria is not considered, they enable one-time simultaneous transfer of resistance and virulence genes into any type of $K$. pneumoniae clones, including cKPs, to facilitate emergence of MDR-HvKPs (Figures 11)B,(1)-C). Indeed, a growing body of evidence supports the hypothesis. Huang et al. (2018) identified a hybrid virulent plasmid which comprised both parts of the pLVPK and an IncHI2-type resistance plasmid in a KPC-2-producing K47 ST11 MDR-HvKP strain in Taiwan. According to the study of Lam et al. (2019), both two ESBL-producing K24 ST15 K. pneumoniae isolates carried large hv-MDR mosaic plasmids which include sequences typical of $\mathrm{IncFIB}_{K}$ virulence plasmids, such as pK2044, fused with regions of homology with typical IncFIIK conjugative MDR plasmids. Similarly, Turton et al. (2019) also described hybrid plasmids containing 
both resistance and virulence clusters in $12 \mathrm{CR}-\mathrm{HvKP}$ isolates belonging to ST15/48/101/147/383. Yet, considering the special characteristics of classic hv plasmid, such as its notable lineage specificity, narrow-host-range, and low-copy-number traits, it might inhibit a second virulent plasmid transfer into the same strain, which thus might cause hvKPs to not acquire and maintain other hybrid plasmids with most hv genes-bearing regions. These surveyed CR-HvKP strains of hvKP-linked STs generally carrying a single hv or MDR-hv hybrid plasmid can support this hypothesis.

Besides, if the In, Tn, and/or Is further carry the virulent sites/genes from the hv plasmid into other resistance plasmids, hybrid plasmids with most sites of the resistance plasmid traits are formed. They can be transferred into either hvKPs (Figure 11-(2)-A) or cKPs (Figure 11-(2)-B) to form MDR-hvKPs mediated by their conjugal transfer system.

From a biological point of view, the highly mosaic nature of antimicrobial resistance and virulence determinants converging within a single vector, the purported co-selection, efficiently facilitates the evolution in two directions, which is a shortcut to an evolutionary success for a proficient bacteria, since selection by relevant antibiotics will also select for virulence traits (Turton et al., 2019). It should be noted that, due to the integration of multiple plasmids elements, including self-transfer conjugative system as well as expanded replicons number and host ranges, hybrid plasmids with MDR and hv biphenotypes can widely spread and infect many types of bacterial hosts and eventually become notorious environmental contaminators (Xie et al., 2020).

\section{CONCLUSION}

In the context of what has already been reported by others regarding the MDR and hv convergence in $K$. pneumoniae, epidemiological characteristics and formation mechanisms of MDR-HvKPs researched in these papers have been discussed and elaborated in the current review.

Collectively, an epidemiology analysis enhances our understanding that the genetic background and geographical distribution characteristics of MDR-HvKP are highly consistent with the epidemic characteristics of hvKP and MDR strains and the monitoring and control of both will help prevent the occurrence of superbugs. The formation mechanism analysis has brought to light that hvKP and MDR strains

\section{REFERENCES}

Andrade, L. N., Vitali, L., Gaspar, G. G., Bellissimo-Rodrigues, F., Martinez, R., and Darini, A. L. (2014). Expansion and evolution of a virulent, extensively drugresistant (polymyxin B-resistant), QnrS1-, CTX-M- 2-, and KPC-2-producing Klebsiella pneumoniae ST11 international high-risk clone. J. Clin. Microbiol. 52, 2530-2535. doi: 10.1128/jcm.00088-14

Arena, F., Henrici, De Angelis, L., D’Andrea, M. M., Cannatelli, A., Fossati, L., et al. (2017). Infections caused by carbapenem-resistant Klebsiella pneumoniae with hypermucoviscous phenotype: A case report and literature review. Virulence 8, 1900-1908. doi: 10.1080/21505594.2017.12 86439 could evolve into MDR-HvKPs through acquiring MDR, hv plasmids, or resistance/virulence hybrid plasmids. The formation mechanisms of biphenotypic composite bacteria are different in distinct genetic background K. pneumoniae clones, but the horizontal transfer of plasmids plays a decisive role. In addition, the adaptive evolution ability of the strain promotes the formation, persistence, and transmission of MDRHvKP.

In the current review, we identified the important role of plasmid-mediated HGT endowing convergence of hv and MDR in K. pneumoniae. A plasmid-centered outlook, as opposed to the traditional host-centric view, should be taken into consideration by clinicians to turn the focal point from the specific host strains to resistance and virulence plasmids (and other MGEs) and to adopt surveillance strategies to track, hinder, or minimize the horizontal dissemination of them. Notably, avoiding drug abuse after the composite strains form may reduce the persistence of plasmids and extreme strains.

\section{AUTHOR CONTRIBUTIONS}

MRT and XK conceived, designed, and wrote the manuscript. JCH performed paper search and graphics visualization. JBL performed writing - reviewing and editing, supervision, and project administration. All authors read and approved the final manuscript.

\section{FUNDING}

This work was supported by grants from (1) Construction of clinical medicine strain resource base and application platform, Sichuan Science and Technology Infrastructure Platform Project (2018TJPT0011) From Science and Technology Department of Sichuan Province, (2) The Study on risk factors and predictive model of high-virulence Klebsiella pneumoniae hepatic abscess, Sichuan International Science and Technology Innovation Cooperation/Hong Kong, Macao and Taiwan Science and Technology Innovation Cooperation Project (2019YFH0021) From Science and Technology Department of Sichuan Province, and (3) Proteomics study of carbapenem resistant Acinetobacter baumannii (14035) from Luzhou Medical College. The funders had no role in study design, data collection and interpretation, or the decision to submit the work for publication.

Bialek-Davenet, S., Criscuolo, A., Ailloud, F., Passet, V., Jones, L., DelannoyVieillard, A.-S., et al. (2014). Genomic Definition of Hypervirulent and Multidrug-Resistant Klebsiella pneumoniae Clonal Groups. Emerg. Infect. Dis. 20, 1812-1820. doi: 10.3201/eid2011.140206

Carlos Catalan-Najera, J., Garza-Ramos, U., and Barrios-Camacho, H. (2017). Hypervirulence and hypermucoviscosity: Two different but complementary Klebsiella spp. phenotypes? Virulence 8, 1111-1123. doi: 10.1080/21505594. 2017.1317412

Cejas, D., Canigia, L. F., Cruz, G. R., Elena, A. X., Maldonado, I., Gutkind, G. O., et al. (2014). First isolate of KPC-2-producing Klebsiella pneumonaie sequence type 23 from the Americas. J. Clin. Microbiol. 52, 3483-3485. doi: 10.1128/jcm. 00726-14 
Cejas, D., Elena, A., Guevara Nunez, D., Sevillano Platero, P., De Paulis, A., Magarinos, F., et al. (2019). Changing epidemiology of KPC-producing Klebsiella pneumoniae in Argentina: Emergence of hypermucoviscous ST25 and high-risk clone ST307. J. Glob. Antimicrob. Resist. 18, 238-242. doi: 10.1016/j. jgar.2019.06.005

Cubero, M., Grau, I., Tubau, F., Pallares, R., Dominguez, M. A., Linares, J., et al. (2016). Hypervirulent Klebsiella pneumoniae clones causing bacteraemia in adults in a teaching hospital in Barcelona. Spain (2007-2013). Clin. Microbiol. Infect. 22, 154-160. doi: 10.1016/j.cmi.2015.09.025

Dong, N., Lin, D., Zhang, R., Chan, E. W., and Chen, S. (2018a). Carriage of blaKPC-2 by a virulence plasmid in hypervirulent Klebsiella pneumoniae. J. Antimicrob. Chemother. 73, 3317-3321. doi: 10.1093/jac/dky358

Dong, N., Sun, Q., Huang, Y., Shu, L., Ye, L., Zhang, R., et al. (2019). Evolution of Carbapenem-Resistant Serotype K1 Hypervirulent Klebsiella pneumoniae by Acquisition of bla $a_{\mathrm{VIM}-1}$-Bearing Plasmid. Antimicrob. Agents Chemother. 63, e01056-e01019. doi: 10.1128/aac.01056-19

Dong, N., Yang, X., Zhang, R., Chan, E. W.C., and Chen, S. (2018b). Tracking microevolution events among ST11 carbapenemase-producing hypervirulent Klebsiella pneumoniae outbreak strains. Emerg. Microb. Infect. 7, 146-153. doi: 10.1038/s41426-018-0146-6

Durao, P., Balbontin, R., and Gordo, I. (2018). Evolutionary Mechanisms Shaping the Maintenance of Antibiotic Resistance. Trends Microbiol. 26, 677-691. doi: 10.1016/j.tim.2018.01.005

Feng, Y., Lu, Y., Yao, Z., and Zong, Z. (2018). Carbapenem-Resistant Hypervirulent Klebsiella pneumoniae of Sequence Type 36. Antimicrob. Agents Chemother. 62, e2644-e2617. doi: 10.1128/aac.02644-17

Frost, L. S., Leplae, R., Summers, A. O., and Toussaint, A. (2005). Mobile genetic elements: the agents of open source evolution. Nat. Rev. Microbiol. 3, 722-732. doi: 10.1038/nrmicro1235

Fu, Y., Xu, M., Liu, Y., Li, A., and Zhou, J. (2019). Virulence and genomic features of a bla(CTX-M-3) and bla(CTX-M-14) coharboring hypermucoviscous Klebsiella pneumoniae of serotype K2 and ST65. Infect. Drug Resist. 12, 145-159. doi: 10.2147/idr.S187289

Gu, D. X., Huang, Y. L., Ma, J. H., Zhou, H. W., Fang, Y., Cai, J. C., et al. (2016). Detection of Colistin Resistance Gene mcr-1 in Hypervirulent Klebsiella pneumoniae and Escherichia coli Isolates from an Infant with Diarrhea in China. Antimicrob. Agents Chemother. 60, 5099-5100. doi: 10.1128/aac.00476- 16

Gu, D., Dong, N., Zheng, Z., Lin, D., Huang, M., Wang, L., et al. (2017). A fatal outbreak of ST11 carbapenem-resistant hypervirulent Klebsiella pneumoniae in a Chinese hospital: a molecular epidemiological study. Lancet Infect Dis. 18, 37-46. doi: 10.1016/s1473-3099(17)30489-9

Gu, D., Lv, H., Sun, Q., Shu, L., and Zhang, R. (2018). Emergence of tet(A) and bla(KPC-2) co-carrying plasmid from a ST11 hypervirulent Klebsiella pneumoniae isolate in patient's gut. Int. J. Antimicrob. Agents 52, 307-308. doi: 10.1016/j.ijantimicag.2018.06.003

Harada, S., Aoki, K., Ishii, Y., Ohno, Y., Nakamura, A., Komatsu, M., et al. (2019). Emergence of IMP-producing hypervirulent Klebsiella pneumoniae carrying a pLVPK-like virulence plasmid. Int. J. Antimicrob. Agents 53, 873-875. doi: 10.1016/j.ijantimicag.2019.05.007

Huang, Y.-H., Chou, S.-H., Liang, S.-W., Ni, C.-E., Lin, Y.-T., Huang, Y.-W., et al. (2018). Emergence of an XDR and carbapenemase-producing hypervirulent Klebsiella pneumoniae strain in Taiwan. J. Antimicrob. Chemother. 73, 20392046. doi: 10.1093/jac/dky164

Lam, M. M. C., Wyres, K. L., Judd, L. M., Wick, R. R., Jenney, A., Brisse, S., et al. (2018). Tracking key virulence loci encoding aerobactin and salmochelin siderophore synthesis in Klebsiella pneumoniae. Genome Med. 10:77. doi: 10. 1186/s13073-018-0587-5

Lam, M. M. C., Wyres, K. L., Wick, R. R., Judd, L. M., Fostervold, A., Holt, K. E., et al. (2019). Convergence of virulence and MDR in a single plasmid vector in MDR Klebsiella pneumoniae ST15. J. Antimicrob. Chemother. 74, 1218-1222. doi: 10.1093/jac/dkz028

Lee, C. R., Lee, J. H., Park, K. S., Jeon, J. H., Kim, Y. B., Cha, C. J., et al. (2017). Antimicrobial Resistance of Hypervirulent Klebsiella pneumoniae: Epidemiology. Hypervirulence-Associated Determinants, and Resistance Mechanisms. Front Cell Infect Microbiol. 7, 483-495. doi: 10.3389/fcimb. 2017.00483

Lee, C. R., Lee, J. H., Park, K. S., Kim, Y. B., Jeong, B. C., and Lee, S. H. (2016). Global Dissemination of Carbapenemase-Producing Klebsiella pneumoniae:
Epidemiology. Genetic Context, Treatment Options, and Detection Methods. Front. Microbiol. 7, 895-924. doi: 10.3389/fmicb.2016.00895

Li, J., Huang, Z.-Y., Yu, T., Tao, X.-Y., Hu, Y.-M., Wang, H.-C., et al. (2019). Isolation and characterization of a sequence type 25 carbapenem-resistant hypervirulent Klebsiella pneumoniae from the mid-south region of China. BMC Microbiol. 19:219-219. doi: 10.1186/s12866-019-1593-5

Li, W., Sun, G. Z., Yu, Y. H., Li, N., Chen, M., Jin, R. H., et al. (2014). Increasing occurrence of antimicrobial-resistant hypervirulent (hypermucoviscous) Klebsiella pneumoniae isolates in China. Clin. Infect. Dis. 58, 225-232. doi: $10.1093 / \mathrm{cid} / \mathrm{cit} 675$

Lin, Y. T., Cheng, Y. H., Juan, C. H., Wu, P. F., Huang, Y. W., Chou, S. H., et al. (2018). High mortality among patients infected with hypervirulent antimicrobial-resistant capsular type K1 Klebsiella pneumoniae strains in Taiwan. Int. J. Antimicrob. Agents 52, 251-257. doi: 10.1016/j.ijantimicag.2018. 06.008

Liu, B., and Su, W. (2019). Whole genome sequencing of NDM-1-producing serotype K1 ST23 hypervirulent Klebsiella pneumoniae in China. J. Med. Microbiol. 68, 866-873. doi: 10.1099/jmm.0.000996

Liu, P. P., Liu, Y., Wang, L. H., Wei, D. D., and Wan, L. G. (2016). Draft Genome Sequence of an NDM-5-Producing Klebsiella pneumoniae Sequence Type 14 Strain of Serotype K2. Genom. Announc. 4, e01610-e01615. doi: 10. 1128/genomeA.01610-15

Liu, Y., Long, D., Xiang, T., Du, F., Wei, D. D., Wan, L. G., et al. (2019). Whole genome assembly and functional portrait of hypervirulent extensively drugresistant NDM-1 and KPC-2 co-producing Klebsiella pneumoniae of capsular serotype K2 and ST86. J. Antimicrob. Chemother. 74, 1233-1240. doi: 10.1093/ $\mathrm{jac} / \mathrm{dkz} 023$

Liu, Z., Gu, Y., Li, X., Liu, Y., Ye, Y., Guan, S., et al. (2019). Identification and Characterization of NDM-1-producing Hypervirulent (Hypermucoviscous) Klebsiella pneumoniae in China. Ann. Lab. Med. 39, 167-175. doi: 10.3343/alm. 2019.39.2.167

Lu, Y., Feng, Y., McNally, A., and Zong, Z. (2018). The Occurence of ColistinResistant Hypervirulent Klebsiella pneumoniae in China. Front. Microbiol. 9, 2568-2574. doi: 10.3389/fmicb.2018.02568

Million-Weaver, S., and Camps, M. (2014). Mechanisms of plasmid segregation: have multicopy plasmids been overlooked? Plasmid 75, 27-36. doi: 10.1016/j. plasmid.2014.07.002

Navon-Venezia, S., Kondratyeva, K., and Carattoli, A. (2017). Klebsiella pneumoniae: a major worldwide source and shuttle for antibiotic resistance. FEMS Microbiol. Rev. 41, 252-275. doi: 10.1093/femsre/ fux013

Pinilla-Redondo, R., Cyriaque, V., Jacquiod, S., Sorensen, S. J., and Riber, L. (2018). Monitoring plasmid-mediated horizontal gene transfer in microbiomes: recent advances and future perspectives. Plasmid. 99, 56-67. doi: 10.1016/j.plasmid. 2018.08.002

Rafiq, Z., Sam, N., and Vaidyanathan, R. (2016). Whole genome sequence of Klebsiella pneumoniae U25, a hypermucoviscous, multidrug resistant, biofilm producing isolate from India. Mem. Inst. Oswaldo Cruz. 111, 144-146. doi: 10.1590/0074-02760150423

Ramsay, J. P., and Firth, N. (2017). Diverse mobilization strategies facilitate transfer of non-conjugative mobile genetic elements. Curr. Opin. Microbiol. 38, 1-9. doi: 10.1016/j.mib.2017.03.003

Roulston, K. J., Bharucha, T., Turton, J. F., Hopkins, K. L., and Mack, D. J. F. (2018). A case of NDM-carbapenemase-producing hypervirulent Klebsiella pneumoniae sequence type 23 from the UK. JMM Case Rep. 5:e005130. doi: 10.1099/jmmcr.0.005130

Schweizer, C., Bischoff, P., Bender, J., Kola, A., Gastmeier, P., Hummel, M., et al. (2019). Plasmid-Mediated Transmission of KPC-2 Carbapenemase in Enterobacteriaceae in Critically Ill Patients. Front. Microbiol. 10:276. doi: 10. 3389/fmicb.2019.00276

Shankar, C., Nabarro, L. E. B., Devanga Ragupathi, N. K., Muthuirulandi Sethuvel, D. P., Daniel, J. L. K., Doss, C. G. P., et al. (2016a). Draft Genome Sequences of Three Hypervirulent Carbapenem-Resistant Klebsiella pneumoniae Isolates from Bacteremia. Genom. Announc. 4, e1081-e1016. doi: 10.1128/genomeA. 01081-16

Shankar, C., Santhanam, S., Kumar, M., Gupta, V., Devanga Ragupathi, N. K., and Veeraraghavan, B. (2016b). Draft Genome Sequence of an ExtendedSpectrum-beta-Lactamase-Positive Hypervirulent Klebsiella pneumoniae Strain 
with Novel Sequence Type 2318 Isolated from a Neonate. Genom. Announc. 4, e1273-e1216. doi: 10.1128/genomeA.01273-16

Shen, D., Ma, G., Li, C., Jia, X., Qin, C., Yang, T., et al. (2019). Emergence of a Multidrug-Resistant Hypervirulent Klebsiella pneumoniae Sequence Type 23 Strain with a Rare bla(CTX-M-24)-Harboring Virulence Plasmid. Antimicrob. Agents Chemother. 63, e2273-e2218. doi: 10.1128/aac.02273-18

Shon, A. S., Bajwa, R. P. S., and Russo, T. A. (2013). Hypervirulent (hypermucoviscous) Klebsiella pneumoniae A new and dangerous breed. Virulence 4, 107-118. doi: 10.4161/viru.22718

Shu, L., Dong, N., Lu, J., Zheng, Z., Hu, J., Zeng, W., et al. (2019). Emergence of OXA-232 Carbapenemase-Producing Klebsiella pneumoniae That Carries a pLVPK-Like Virulence Plasmid among Elderly Patients in China. Antimicrob. Agents Chemother. 63, e2246-e2218. doi: 10.1128/aac.02246-18

Smillie, C., Garcillán-Barcia, M. P., Francia, M. V., Rocha, E. P. C., and de la Cruz, F. (2010). Mobility of plasmids. Microbiol. Mol. Biol. Rev. 74, 434-452. doi: 10.1128/MMBR.00020-10

Surgers, L., Boyd, A., Girard, P.-M., Arlet, G., and Decre, D. (2016). ESBLProducing Strain of Hypervirulent Kiebsiella pneumoniae K2. France. Emerg. Infect. Dis. 22, 1687-1688. doi: 10.3201/eid2209.160681

Tabrizi, A. M. A., Badmasti, F., Shahcheraghi, F., and Azizi, O. (2018). Outbreak of hypervirulent Klebsiella pneumoniae harbouring bla(VIM-2) among mechanically-ventilated drug-poisoning patients with high mortality rate in Iran. J. Glob. Antimicrob. Resist. 15, 93-98. doi: 10.1016/j.jgar.2018. 06.020

Turton, J. F., Payne, Z., Coward, A., Hopkins, K. L., Turton, J. A., Doumith, M., et al. (2017). Virulence genes in isolates of Klebsiella pneumoniae from the UK during 2016, including among carbapenemase gene-positive hypervirulent K1ST23 and 'non-hypervirulent'types ST147, ST15 and ST383. J. Med. Microbiol. 67, 118-128. doi: 10.1099/jmm.0.000653

Turton, J., Davies, F., Turton, J., Perry, C., Payne, Z., and Pike, R. (2019). Hybrid Resistance and Virulence Plasmids in "High-Risk". Clones of Klebsiella pneumoniae, Including Those Carrying blaNDM-5. Microorganisms 7, 326-336. doi: 10.3390/microorganisms7090326

Tzouvelekis, L. S., Markogiannakis, A., Psichogiou, M., Tassios, P. T., and Daikos, G. L. (2012). Carbapenemases in Klebsiella pneumoniae and other Enterobacteriaceae: an evolving crisis of global dimensions. Clin. Microbiol. Rev. 25, 682-707. doi: 10.1128/CMR.05035-11

Wei, D. D., Wan, L. G., and Liu, Y. (2018). Draft Genome Sequence of an NDM1- and KPC-2-Coproducing Hypervirulent Carbapenem-Resistant Klebsiella pneumoniae Strain Isolated from Burn Wound Infections. Genom. Announc. 6, e118-e192. doi: 10.1128/genomeA.00192-18

Wei, D. D., Wan, L. G., Deng, Q., and Liu, Y. (2016). Emergence of KPC-producing Klebsiella pneumoniae hypervirulent clone of capsular serotype K1 that belongs to sequence type 11 in Mainland China. Diagn. Microbiol. Infect. Dis. 85, 192-194. doi: 10.1016/j.diagmicrobio.2015.03.012

Wong, M. H. Y., Shum, H.-P., Chen, J. H. K., Man, M.-Y., Wu, A., Chan, E. W.C., et al. (2018). Emergence of carbapenem-resistant hypervirulent Klebsiella pneumoniae. Lancet Infect. Dis. 18, 24-25. doi: 10.1016/s1473-3099(17) 30629-1

Wyres, K. L., Lam, M. M. C., and Holt, K. E. (2020). Population genomics of Klebsiella pneumoniae. Nat. Rev. Microbiol. 18, 344-359. doi: 10.1038/s41579019-0315-1

Wyres, K. L., Wick, R. R., Judd, L. M., Froumine, R., Tokolyi, A., Gorrie, C. L., et al. (2019). Distinct evolutionary dynamics of horizontal gene transfer in drug resistant and virulent clones of Klebsiella pneumoniae. PLoS Genet. 15, e1008114-e1008138. doi: 10.1371/journal.pgen.1008114

Xie, M., Chen, K., Ye, L., Yang, X., Xu, Q., Yang, C., et al. (2020). Conjugation of Virulence Plasmid in Clinical Klebsiella pneumoniae Strains through Formation of a Fusion Plasmid. Adv. Biosyst. 4:e1900239. doi: 10.1002/adbi.2019 00239

Xie, Y., Tian, L., Li, G., Qu, H., Sun, J., Liang, W., et al. (2018). Emergence of the third-generation cephalosporin-resistant hypervirulent Klebsiella pneumoniae due to the acquisition of a self-transferable blaDHA-1-carrying plasmid by an ST23 strain. Virulence 9, 838-844. doi: 10.1080/21505594.2018.1456229

Xu, M., Fu, Y. Q., Fang, Y. H., Xu, H., Kong, H. S., Liu, Y. C., et al. (2019). High prevalence of KPC-2-producing hypervirulent Klebsiella pneumoniae causing meningitis in Eastern China. Infect. Drug Resist. 12, 641-653. doi: 10.2147/idr. S191892

Xu, M., Li, A., Kong, H. S., Zhang, W. L., Chen, H. C., Fu, Y. J., et al. (2018). Endogenous endophthalmitis caused by a multidrug-resistant hypervirulent Klebsiella pneumoniae strain belonging to a novel single locus variant of ST23: first case report in China. BMC Infect. Dis. 18, 669-674. doi: 10.1186/s12879018-3543-5

Yao, B., Xiao, X. M., Wang, F., Zhou, L., Zhang, X. W., and Zhang, J. (2015). Clinical and molecular characteristics of multi-clone carbapenem-resistant hypervirulent (hypermucoviscous) Klebsiella pneumoniae isolates in a tertiary hospital in Beijing. China. Int. J. Infect. Dis. 37, 107-112. doi: 10.1016/j.ijid.2015. 06.023

Yao, H., Qin, S., Chen, S., Shen, J., and Du, X. D. (2018). Emergence of carbapenemresistant hypervirulent Klebsiella pneumoniae. Lancet Infect Dis. 18:25. doi: 10.1016/s1473-3099(17)30628-X

Yuan, Y., Li, Y., Wang, G., Li, C., Chang, Y.-F., Chen, W., et al. (2019). bla(NDM5) carried by a hypervirulent Klebsiella pneumoniae with sequence type 29. Antimicrob. Resist. Infect. Control. 8, 142-149. doi: 10.1186/s13756-019-0596-1

Zhan, L. L., Wang, S. S., Guo, Y. J., Jin, Y., Duan, J. J., Hao, Z. H., et al. (2017). Outbreak by Hypermucoviscous Klebsiella pneumoniae ST11 Isolates with Carbapenem Resistance in a Tertiary Hospital in China. Front. Cell Infect. Microbiol. 7, 182-190. doi: 10.3389/fcimb.2017.00182

Zhang, J., Zhou, K., Zheng, B. W., Zhao, L. N., Shen, P., Ji, J. R., et al. (2016). High Prevalence of ESBL-Producing Klebsiella pneumoniae Causing CommunityOnset Infections in China. Front. Microbiol. 7, 1830-1839. doi: 10.3389/fmicb. 2016.01830

Zhang, R., Lin, D. C., Chan, E. W. C., Gu, D. X., Chen, G. X., and Chen, S. (2015). Emergence of Carbapenem-Resistant Serotype K1 Hypervirulent Klebsiella pneumoniae Strains in China. Antimicrob. Agents Chemother. 60, 709-711. doi: 10.1128/aac.02173-15

Zhang, Y. W., Zeng, J., Liu, W. E., Zhao, F., Hu, Z. D., Zhao, C. J., et al. (2015). Emergence of a hypervirulent carbapenem-resistant Klebsiella pneumoniae isolate from clinical infections in China. J. Infect. 71, 553-560. doi: 10.1016/j. jinf.2015.07.010

Zhang, Y. W., Zhao, C. J., Wang, Q., Wang, X. J., Chen, H. B., Li, H. A., et al. (2016). High Prevalence of Hypervirulent Klebsiella pneumoniae Infection in China: Geographic Distribution, Clinical Characteristics, and Antimicrobial Resistance. Antimicrob. Agents Chemother. 60, 6115-6120. doi: 10.1128/aac. 01127-16

Conflict of Interest: The authors declare that the research was conducted in the absence of any commercial or financial relationships that could be construed as a potential conflict of interest.

Copyright (C) 2020 Tang, Kong, Hao and Liu. This is an open-access article distributed under the terms of the Creative Commons Attribution License (CC BY). The use, distribution or reproduction in other forums is permitted, provided the original author(s) and the copyright owner(s) are credited and that the original publication in this journal is cited, in accordance with accepted academic practice. No use, distribution or reproduction is permitted which does not comply with these terms. 